\title{
Atorvastatin induces autophagic cell death in prostate cancer cells in vitro
}

\author{
ZHENHUA HE ${ }^{1-3^{*}}$, JINGMIN YUAN $^{4 *}$, PING QI $^{2}$, LIXIU ZHANG ${ }^{2}$ and ZHIPING WANG ${ }^{1,2}$ \\ ${ }^{1}$ School of Life Sciences, Lanzhou University, Lanzhou, Gansu 730000; \\ ${ }^{2}$ Institute of Urology and Key Laboratory of Urological Diseases in Gansu, The Second Hospital of Lanzhou University; \\ Departments of ${ }^{3}$ Neurosurgery and ${ }^{4}$ Pain, The Second Hospital of Lanzhou University, Lanzhou, Gansu 730030, P.R. China
}

Received May 5, 2014; Accepted January 23, 2015

DOI: $10.3892 / \mathrm{mmr} .2015 .3334$

\begin{abstract}
Athough it is well known that apoptosis contributes to cancer cell death, the role of autophagy in cancer cell death has remained in dispute. Atorvastatin has been suggested to exhibit anti-cancer effects. The present study aimed to examine atorvastatin-induced autophagy-associated cell death and the autophagy-associated gene expression profile in the PC3 prostate carcinoma cell line. The atorvastatin-induced process of autophagy in PC3 cells was determined via evaluation of the cellular expression levels of autophagosomal marker light-chain-3 (LC3)-II, using immunoblotting and counting of green fluorescent protein (GFP)-LC3-transfected autophagic cells. Apoptosis was examined by terminal deoxynucleotidyl transferase dUTP nick end labeling assay and an MTT assay was used to evaluate cell viability. Total RNA of PC3 cells was isolated for characterization of the gene expression profile following atorvastatin treatment. Atorvastatin treatment of PC3 cells for $24 \mathrm{~h}$ increased the expression of green fluorescent protein-LC3-II by $>25 \%$, and expression continued for $>72 \mathrm{~h}$, while apoptosis was not significantly induced within this time period. Four genes associated with the autophagy machinery were also significantly upregulated. In the presence of atorvastatin, autophagy may be unable to abrogate cell damage and may therefore contribute to cellular dysfunction, leading to autophagic/type II programmed cell death. In response to atorvastatin treatment, the expression of genes involved in autophagic mediating pathways may have a role in tumor suppression.
\end{abstract}

Correspondence to: Dr Zhiping Wang, Institute of Urology and Key Laboratory of Urological Diseases in Gansu, The Second Hospital of Lanzhou University, 82 Cuiyingmen, Chengguan, Lanzhou, Gansu 730030, P.R. China

E-mail:erywzp@lzu.edu.cn

${ }^{*}$ Contributed equally

Key words: autophagy, apoptosis, atorvastatin, gene expression

\section{Introduction}

Prostate cancer is the most frequently diagnosed non-cutaneous malignancy and the second leading cause of death due to cancer amongst males worldwide (1). Treatment options for localized disease include watchful waiting, surgery and radiotherapy (2). However, in terms of a definitive treatment, despite developments in systemic chemotherapy strategies, minimal improvements in the quality of life and overall survival have been achieved amongst patients with prostate cancer (3). The development of novel treatment strategies for patients with advanced metastatic prostate cancers remains a challenge.

The PC3 human prostate cancer cell line [p53- and phosphatase and tensin homolog (PTEN)-] was established from a prostatic adenocarcinoma, which was metastatic to bone. It has been extensively used as a cell model for the study of prostate cancer and is generally assumed to model an advanced stage of prostate cancer (4). PC3 cells are resistant to numerous chemotherapy drugs and apoptosis inducers $(5,6)$.

Atorvastatin, a 3-hydroxy-3-methylglutaryl (HMG)-coenzyme A reductase inhibitor, is among the widely prescribed drugs used to lower cholesterol and prevent cardiovascular diseases (7). In addition to its cholesterol-lowering effect, atorvastatin has pro-apoptotic and anti-metastatic effects on prostate cancer cells $(8,9)$. Parikh et al (10) hypothesized that atorvastatin may induce autophagy-associated cell death in PC3 cells. However, the biological mechanisms underlying the anti-cancer effects of atorvastain remain to be elucidated.

Autophagy is a homeostatic, catabolic process responsible for the packaging and degradation of cytoplasmic proteins and organelles. Autophagy is therefore required in order to maintain genomic stability and overall cell survival (11). During autophagosome formation, light-chain-3 (LC3), a cytosolic microtubule-associated protein, is conjugated to phosphatidylethanolamine to form LC3-II prior to its insertion into the autophagosome membrane (12). Evaluation of the translocation and processing of enhanced green fluorescent protein (eGFP)-LC3 fusion proteins is frequently utilized as a marker of autophagic activity (13).

Complex associations exist between the processes of autophagy and apoptosis. Apoptosis and autophagy are 
genetically modulated, evolutionarily conserved processes responsible for the regulation of cell fate (14). However, whereas apoptosis directly induces cancer cell death, autophagy functions as a survival response to growth factor or nutrient deprivation, as well as being a molecular mechanism for tumor-cell death (15). Numerous studies have suggested that if cellular damage is extensive, or if apoptosis is compromised, cell death may be induced via autophagy $(16,17)$.

Multiple autophagy-associated genes have been selected for study, because they are not only required for autophagy, but also as tumor suppressors under certain conditions. For example, the beclinl gene, which induces autophagy when overexpressed in mammalian cells, also functions as a tumor suppressor in human cancer $(18,19)$.

Multiple drugs have been developed to target the process of autophagy in an attempt to manipulate it into an anti-cancer pathway $(20,21)$. The present study aimed to evaluate the effect of atorvastatin treatment on genes involved in the initiation or activation of autophagy and apoptotic signaling pathways. The results of the present study indicate potential treatment combinations to augment the effects of atorvastatin. Super-additive effects of atorvastatin treatment may be achieved by use of atorvastatin in combination with regulation of autophagy-associated genes or co-regulation of autophagic and apoptotic genes to accelerate autophagic cell death in PC3 cells.

\section{Materials and methods}

Cell culture. PC3 prostate carcinoma cells were purchased from the American Type Culture Collection (Manassas, VA, USA) and maintained in GluMax MEM with $10 \%$ fetal bovine serum, $1 \%$ non-essential amino acid and $1 \%$ penicillin/streptomycin, which were all purchased from Invitrogen Life Technologies (Carlsbad, CA, USA), in $37^{\circ} \mathrm{C}$ humidified incubator supplied with $5 \% \mathrm{CO}_{2}$.

pCMV expression vectors containing green fluorescent protein (GFP) or GFP-tagged microtubule-associated protein 1 LC3 (GFP-LC3) were purchased from Cell Biolabs (San Diego, CA, USA). Atorvastatin was purchased from Sigma-Aldrich (St. Louis, MO, USA). The LC3-II purified monoclonal antibody was purchased from Abgent (San Diego, CA, USA; AM1800a). Primary monoclonal antibodies against human GAPDH (ab8245) and secondary donkey anti-rabbit (NA934)/mouse (N1034) immunoglobulin G antibodies were obtained from GE Healthcare Life Sciences (Uppsala, Sweden). An enhanced chemoluminescence (ECL) Plus kit was obtained from GE Healthcare Life Sciences.

GFP-LC3 assay. A GFP-tagged LC3 assay was used to detect and quantify the induction of autophagy, since constitutively expressed LC3-II is recruited to the autophagosomal membrane during autophagy. PC3 cells (90\% confluent) were transiently transfected with the GFP-LC3 vector or GFP control vector using Lipofectamine ${ }^{\circledR} 2000$ reagent (Invitrogen Life Technologies). The transfection rate was determined using flow cytometry (Guava Flow Cytometry system, 0500-4005; Millipore, Billerica, MA, USA) which revealed 60-70\% GFP-positive cells with $2 \times 10^{5}$ cells/U, and following $48 \mathrm{~h}$ incubation cells were treated with atorvastatin $(6 \mu \mathrm{g} / \mathrm{ml}$; Sigma-Aldrich). Following
24, 48 and $72 \mathrm{~h}$ of treatment, cells were washed once with cold phosphate-buffered saline (PBS; Boshide Biotechnology Co., Ltd., Wuhan, China), fixed with $4 \%$ paraformaldehyde, counterstained with DAPI (Sigma-Aldrich) and examined under a fluorescence microscope and a confocal laser scanning microscope (TCS SP8; Leica, Wetzlar, Germany). A diffuse distribution of GFP-LC3 is expressed in the cells under control conditions, whereas a punctate pattern of GFP-LC3 expression is presented in those cells that undergo autophagy. A total of 600 GFP-positive cells from three regions of each sample slide were evaluated and the number of autophagic cells that demonstrated GFP-LC3 puncta ( $\geq 4$ puncta/cell) was determined.

Western blot analysis. Cells were treated with atorvastatin $(6 \mu \mathrm{g} / \mathrm{ml})$, and at $28 \mathrm{~h}$ of treatment, cells were washed with ice-cold PBS and the lysate was isolated using M-PER Mammalian Protein Extraction reagent (Thermo Fisher Scientific, Waltham, MA, USA). Equivalent amounts of cell lysate protein $(50 \mu \mathrm{g})$ were separated by gel electrophoresis on a 4-20\% gradient SDS-PAGE gel (GE Healthcare, Logan, UT, USA), transferred onto nitrocellulose membranes (Bioleaf Biotech, Shanghai, China). Membranes were blocked with $5 \%$ non-fat milk and then incubated with anti-human LC3-II (1:400) or anti-human GAPDH antibodies $(1: 5,000)$ at $4^{\circ} \mathrm{C}$ overnight. Membranes were then washed with $0.1 \%$ Tween 20 in PBS and probed with horseradish-peroxidase conjugated donkey anti-rabbit antibodies $(1: 1,000)$ and donkey anti-mouse antibodies $(1: 10,000)$ for $1 \mathrm{~h}$ at room temperature. The bound antibody complexes were detected using an ECL Plus kit using a Storm 840 PhosphorImager system (Molecular Devices, Sunnyvale, CA, USA). The image was further analyzed using ImageQuant TL 8.1 software (GE Healthcare Life Sciences).

MTT assay. Cell viability was assessed at 24, 32, 48 and $72 \mathrm{~h}$ of atorvastatin $(6 \mu \mathrm{g} / \mathrm{ml})$ treatment. Cell damage was quantified using a tetrazolium salt colorimetric assay with MTT (Sigma-Aldrich). Briefly, MTT was added to each well with $1 \times 10^{3}-1 \times 10^{4}$ cells (final concentration, $1 \mathrm{mg} / \mathrm{ml}$ ) at the indicated time and incubated for $4 \mathrm{~h}$ at $37^{\circ} \mathrm{C}$. The dye was subsequently solubilized with dimethyl sulfoxide (DMSO; $100 \mu \mathrm{l}$; Sigma-Aldrich), and the absorbance of each sample was measured at $490 \mathrm{~nm}$ in a 96-well plate-reader (SpectraMax Plus 384; Molecular Devices). The absorbance of cells without MTT dye was subtracted from each value. The data were normalized to the absorbance of vehicle-treated cells (100\%) and expressed as a percentage of inhibition. Results were obtained from $n=3$ wells per experiment from two separate experiments.

Terminal deoxynucleotidyl transferase dUTP nick end labeling (TUNEL) assay. Cells were divided into three groups according to the different treatments (untreated, $6 \mu \mathrm{g} / \mathrm{ml}$ atorvastatin or $5 \mu \mathrm{l} \mathrm{DMSO}$ ). All three groups were then labeled with Guava Annexin V reagent (Millipore) and fixed with 4\% paraformaldehyde (Sigma-Aldrich) for using in the TUNEL assay (QIA33; Millipore) according to the manufacturer's protocol following 24, 48 and $72 \mathrm{~h}$ treatment. Cells were treated with atorvastatin $(6 \mu \mathrm{g} / \mathrm{ml})$ and DMSO (5 $\mu \mathrm{l})$, at 24, 48 and $72 \mathrm{~h}$, detached using trypsin-EDTA and 
A

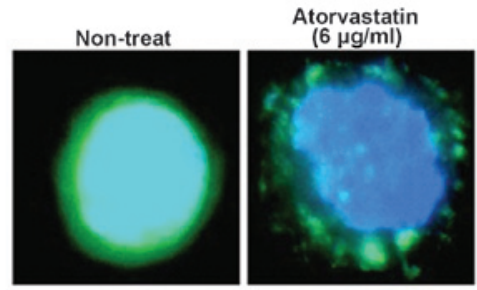

B

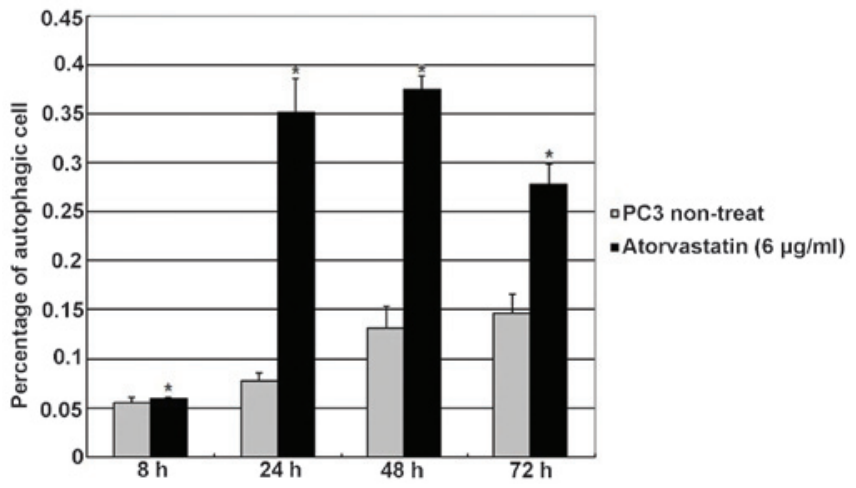

Figure 1. (A) Images of a control cell and an autophagic cell induced by atorvastatin treatment. A representative image (magnification, x630) of PC3 prostate cancer cells revealed that the characteristic punctate appearance of GFP-LC3 expression, indicative of autophagosome formation, increased following atorvastatin treatment. (B) Quantitative comparison of the percentage of autophagic cells between the treatment and control groups. GFP-LC3 transfection studies indicated that atorvastatin promoted autophagosome formation between 24 and $72 \mathrm{~h}$ of treatment in PC3 cells Values are presented as the mean \pm standard for each sample slide $(n=3)$. ${ }^{*} \mathrm{P}<0.05$ vs. control group. PC3 non-treat, control group; GFP, green fluorescent protein; LC3, light-chain-3.

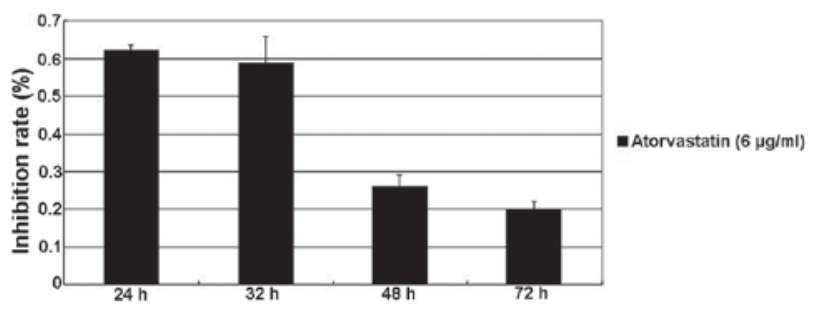

Figure 2. Comparison of the MTT reduction rate between the two groups. There was marked inhibition of cell proliferation $24 \mathrm{~h}$ following atorvastatin treatment compared with that of the control (dimethyl sulfoxide-treated) group.

fixed with $4 \%$ paraformaldehyde for TUNEL assay according to the manufacturer's instructions. Cells were subsequently analyzed using a Guava Personal Cell Analysis (PCA) system (EMD Millipore).

$R T^{2}$ profiler polymerase chain reaction (PCR) array. Atorvastatin $(6 \mu \mathrm{g} / \mathrm{ml})$ and DMSO $(5 \mu \mathrm{l})$ were added into preseeded cells. Following $28 \mathrm{~h}$ of incubation, cells ( $1 \times 10^{5} /$ well) were washed with cold PBS, total RNA was isolated using an RNeasy kit and treated with DNase A (Qiagen, Valencia, CA, USA). The cDNA was synthesized from $1 \mu \mathrm{g}$ total RNA using an $\mathrm{RT}^{2} \mathrm{PCR}$ array first strand kit (Qiagen). The $\mathrm{RT}^{2}$ Profiler PCR array for the autophagy pathway (Qiagen) was subsequently analyzed using a Bio-Rad CFX96 real-time PCR instrument (Bio-Rad Laboratories, Inc., Hercules, CA, USA) according to the manufacturer's instructions, in order to

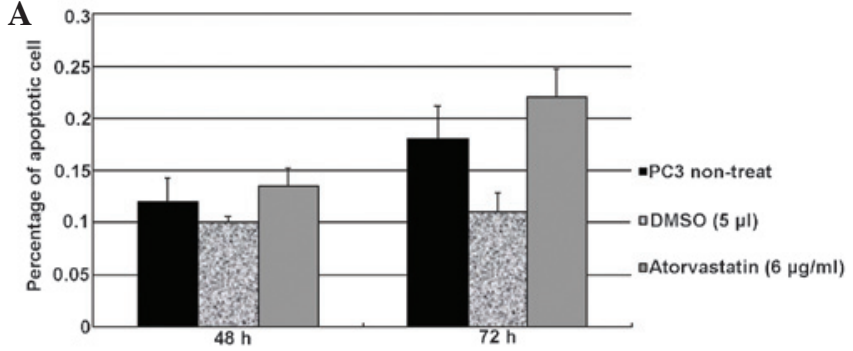

B
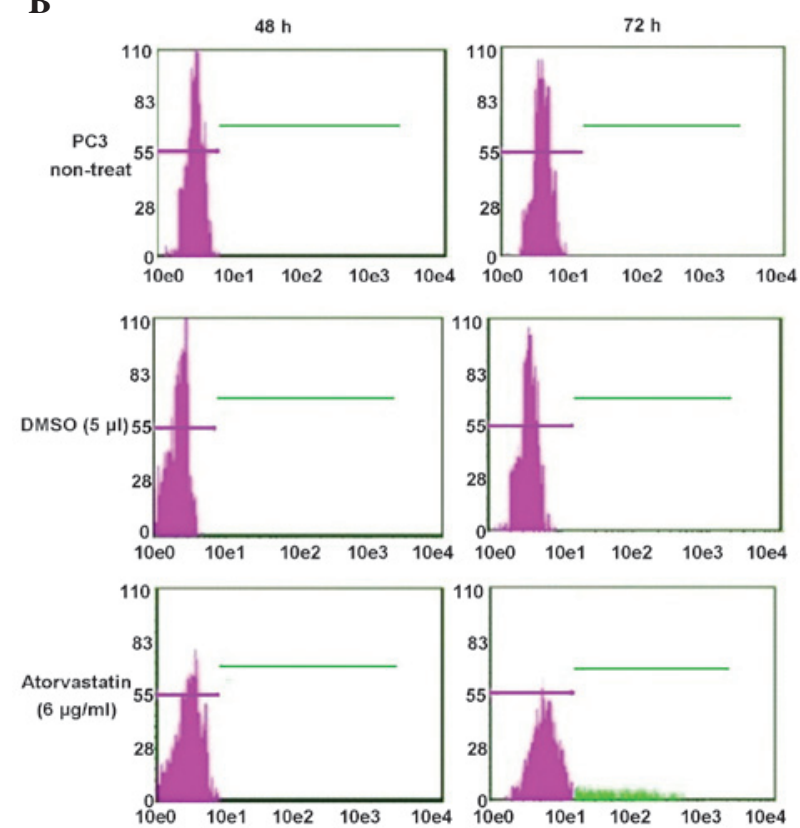

Figure 3. Comparison of the percentage of apoptotic cells between the three groups. (A) Apoptotic rate as determined by quantification of TUNEL staining. (B) Flow cytometric analysis of TUNEL staining. Low levels of apoptosis occurred prior to $72 \mathrm{~h}$ post-atorvastatin treatment in PC3 cells. TUNEL, terminal deoxynucleotidyl transferase dUTP nick end labeling.

examine the expression of 84 genes. The fold-change in gene expression was calculated using the Excel-based PCR Array Data Analysis program (version 3.5; Qiagen).

Statistical analysis. All statistical analyses were performed using the Excel Data Analysis program (Microsoft Corp., Redmond, WA, USA). Two individual groups were evaluated by Student's t-test and $\mathrm{P}<0.05$ was considered to indicate a statistically significant difference.

\section{Results}

Atorvastatin treatment activates autophagy in PC3 cells. The activation of the autophagic process in PC 3 cells by atorvastatin was determined using a GFP-LC3 assay, in which the number of autophagic cells was counted using previously described criteria ( $\geq 4$ GFP-LC3 puncta/cell indicated autophagic cells; Fig. 1A) (22). The results revealed a significantly increased number of autophagic cells (35\% of transfected cells) $24 \mathrm{~h}$ after atorvastatin incubation compared with that of the control group (8\%). The number of autophagic cells peaked at $48 \mathrm{~h}$ of treatment, reaching $38 \%$ of transfected cells. This peak was followed by a slight decrease in the percentage of autophagic cells at $72 \mathrm{~h}$ of treatment. (Fig. 1B). 
Table I. Expression changes of autophagy-associated genes and autophagy-apoptosis co-regulated genes at $28 \mathrm{~h}$ post-atorvastatin treatment.

Atorvastatin $(6 \mu \mathrm{g} / \mathrm{ml})$

Gene

Fold change P-value

Autophagy machinery components

$\begin{array}{lrr}\text { GabarapL1 } & 4.52 & 0.030 \\ \text { MAP1LC3A } & 4.55 & 0.010 \\ \text { MAP1LC3B } & 2.14 & 0.040 \\ \text { ULK1 } & 10.64 & 0.100\end{array}$

Co-regulators of autophagy and apoptosis

$\begin{array}{lll}\text { ARF } & 2.29 & 0.009 \\ \text { TMEM74 } & 3.93 & 0.050 \\ \text { Arsa } & 6.59 & 0.100 \\ \text { Bnip3 } & 1.98 & 0.007 \\ \text { Eif2ak3 } & 4.67 & 0.005 \\ \text { Prkaa1 } & 1.87 & 0.040\end{array}$

A

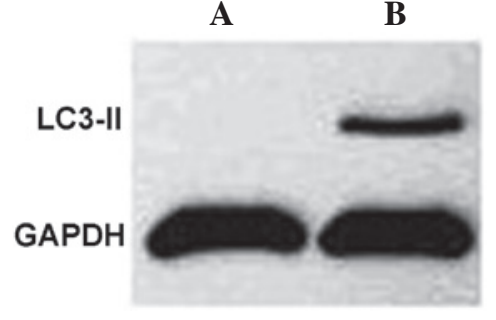

Figure 4. Formation of LC3-II protein in atorvastatin-treated PC3 cells. (A) Non-treated control group. (B) LC3-II formation was induced at $24 \mathrm{~h}$ post-atorvastatin treatment $(6 \mu \mathrm{g} / \mathrm{ml}, 28 \mathrm{~h})$. LC3, light-chain-3.

Atorvastatin reduces cell viability. Atorvastatin has been shown to inhibit cancer cell proliferation (23). Consistent with these observations, the results of the present study revealed a marked inhibition of cell proliferation from $24 \mathrm{~h}$ of treatment (Fig. 2). There was little apoptosis occurring prior to $72 \mathrm{~h}$ with atorvastatin treatment in PC3 cells (Fig. 3). Conversely, atorvastatin was able to induce autophagy within 24-72 $\mathrm{h}$ (Fig. 1B), raising the possibility that autophagy may function in the induction of type-II cell death following atorvastatin treatment in PC3 cells.

Atorvastatin treatment induces LC3II formation and apoptosis. A TUNEL assay was performed to assess the level of apoptosis, and the results indicated that no significant apoptosis occurred within the 24-72 h time-period (Fig. 3). Western blot analysis revealed that formation of LC3II, an autophagy marker, was induced at $24 \mathrm{~h}$ of atorvastatin treatment in the treatment group but not in control cells (Fig. 4).

Gene expression profiles of autophagy. To investigate the factors underlying the induction of autophagy in PC3 cells following atorvastatin treatment, the expression profiles of autophagy-associated genes were compared between atorvastatin and DMSO-treated groups using the PCR array technique. The PCR array panel used in the present study contained 84 genes which are involved in the autophagic machinery and regulation. Among these genes, four autophagy machinery genes (GABARAPL1, ULK1, MAP1LC3A and MAP1LC3B) were found to be upregulated ( $\geq 2$-fold). Of note, multiple genes which co-regulate autophagy and apoptosis were also found to exhibit a marked increase in expression levels in atorvastatin-treated PC3 cells (Table I).

\section{Discussion}

Atorvastatin, also known under the brand name Lipitor, is a cholesterol-reducing drug which inhibits cholesterol synthesis (24). Decreasing endogenous levels of cholesterol may aid the prevention of heart disease and atherosclerosis, conditions which potentially lead to heart attack, stroke and/or vascular diseases (25). It was reported several years ago that atorvastatin inhibited cancer growth and induced cancer cell death. However, the specific genes and signal transduction cascade underlying the anti-tumor effects of atorvastatin had remained elusive.

Consistent with the results of previous studies, atorvastatin was shown to induce autophagy and apoptosis in PC3 cells (26), but a time interval between the induction of autophagy and apoptosis was also identified. The results of the present study demonstrated that PC3 cells began to exhibit characteristics of autophagy $\sim 24 \mathrm{~h}$ of treatment and remained in the autophagic state until $72 \mathrm{~h}$ of treatment, after which apoptosis was observed. It was also demonstrated that atorvastatin inhibited cell proliferation between 24-72 h of treatment. It was hypothesized that autophagy may be a catabolic process involved in the maintenance of homeostasis via the elimination of superfluous proteins and damaged organelles (27). However, in the presence of atorvastatin, autophagy may be unable to reverse the damage and therefore induce cellular dysfunction, resulting in autophagic/type II programmed cell death. 
It is well-known that highly expressed genes result in higher constitutive expression of the encoded protein, which may influence the further response of cancer cells to chemo/radiotherapy (28). In the present study, the expression of 84 genes involved in autophagy pathways was investigated using a PCR array in order to evaluate their participation in the autophagic process and thereby identify genes that may be capable of enhancing the anti-tumor effect of atorvastatin. These 84 genes may be categorized into autophagic machinery components and autophagy regulators. Autophagy was initially identified as a cellular survival response to starvation; however, an increasing body of evidence has indicated that this process may also be induced by various stress conditions, including chemotherapy and radiotherapy $(29,30)$. However, whether autophagy is a cell death mechanism or a survival mechanism remains to be elucidated. Improving treatment efficiency requires the development of more specific therapies, based on distinct gene expression patterns.

The early stages of autophagosome formation require ULK1 (the ortholog of Atg1 kinase), the PtdIns-3 kinase Vps34 and two ubiquitin-like conjugation systems composed of the ubiquitin-like protein LC3-I, an ortholog of yeast Atg8, and the Atg 4 protease, the functions of which result in the formation of the Atg5-12-16 complex and LC3-II (31). LC3-II is a lipid-conjugated form of LC3-I, and is the most frequently used marker of autophagy. This core machinery drives the initiation, expansion and closure of the isolation membrane. In the present study, it was revealed that ULK1 expression was increased 10.41-fold suggesting that it is a significant gene involved in the induction of autophagy following atorvastatin treatment.

The late stage of the autophagic response comprises the delivery of the contents sequestered by the autophagosome to lysosomes for degradation (32). TMEM74 modulates the formation of autophagolysosomes and subsequently accelerates functional autophagy (33). The results of the present study suggested that TMEM74 was a key gene involved in the atorvastatin-induced autophagy process, due to its overexpression following atorvastatin treatment.

GABARAPL1 and MAP1LC3B are mammalian autophagosomal orthologs of the yeast Atg8 gene, which is associated with the formation of the double-membraned autophagic vacuole $(34,35)$. The results of the present study revealed overexpression of these genes (4.52- and 2.14-fold, respectively), which suggested that these two autophagy-associated genes also participate in the atorvastatin-induced autophagy process. An increasing body of evidence indicated that complex interactions may exist between the autophagic and apoptotic cell death pathways (36). Excessive levels of autophagy may be associated with the activation of apoptosis via an autodigestive pathway $(37,38)$. Espert et al (39) hypothesized that autophagy may function upstream of certain signaling transduction events which lead to apoptotic cell death. Chen et al (40) demonstrated that MAP1LC3B-/-mice had significantly decreased levels of apoptosis in vivo. Conversely, apoptotic cell death was significantly increased in MAP1LC3B $+/+$ mice. In the present study, MAP1LC3B exhibited a $>2$-fold increase in expression following atorvastatin treatment. It was therefore hypothesized that the late apoptosis resulting from atorvastatin treatment after $72 \mathrm{~h}$ may occur as a result of activated autophagy and autophagy-induced cell death.
PC3 cells are p53-null, and it was previously demonstrated that $\mathrm{p} 19(\mathrm{ARF})$ possesses a p53-independent function in tumor suppression. The level of incidence and aggression of tumors in p53/ARF double knock-out mice are greater than those in p53 knock-out mice (41). Furthermore, overexpression of ARF has been shown to repress p53-null cell proliferation $(42,43)$. In the present study, it was suggested that atorvastatin may induce autophagy-associated cell death in PC3 cells. The results revealed increased expression (2.16-fold) of ARF, following atorvastatin treatment. There are compelling data to suggest that autophagy frequently has a role in tumor suppression $(19,44)$. Based on these results, it was hypothesized that ARF may be a significant cancer suppressor gene upon atorvastatin treatment in PC3 cells.

In conclusion, the present study demonstrated that atorvastatin treatment significantly influenced the cell viability of PC3 cells. The results suggested that atorvastatin may induce cell death in PC3 cells via an autophagic cell death pathway. Furthermore, to the best of our knowledge, the present study was the first to detect the autophagy-associated gene expression profile induced by atorvastatin treatment. The response of cancer patients to atorvastatin may be dependent on the signaling context that is generated by autophagy-associated gene expression levels in the PC3 prostate cancer cell line. Further investigation is required to focus on the regulation of autophagy-associated genes in order to enhance the anti-tumor effects of atorvastatin treatment.

\section{Acknowledgements}

The present study was supported by the China Postdoctoral Science Foundation funded project (2013M542395) and Natural Science Foundation Project of Gansu province (145RJYA256).

\section{References}

1. Siegel R, Naishadham D and Jemal A: Cancer statistics, 2012. CA Cancer J Clin 62: 10-29, 2012.

2. Siegel R, DeSantis C, Virgo K, et al: Cancer treatment and survivorship statistics, 2012. CA Cancer J Clin 62: 220-241, 2012.

3. Sun F, Oyesanmi O, Fontanarosa J, Reston J, Guzzo T and Schoelles K: Therapies for clinically localized prostate cancer: Update of a 2008 systematic review. AHRQ Comparative Effectiveness Reviews: 15-EHC004-EF, 2014.

4. Dozmorov MG, Hurst RE, Culkin DJ, et al: Unique patterns of molecular profiling between human prostate cancer $\mathrm{LNCaP}$ and PC-3 cells. Prostate 69: 1077-1090, 2009.

5. An J, Chervin AS, Nie A, Ducoff HS and Huang Z: Overcoming the radioresistance of prostate cancer cells with a novel Bcl-2 inhibitor. Oncogene 26: 652-661, 2007.

6. Marcelli M, Marani M, Li X, et al: Heterogeneous apoptotic responses of prostate cancer cell lines identify an association between sensitivity to staurosporine-induced apoptosis, expression of $\mathrm{Bcl}-2$ family members and caspase activation. Prostate 42: 260-273, 2000

7. Lewington S, Whitlock G, Clarke R, Sherliker P, Emberson J, Halsey J, Qizilbash N, Peto R and Collins R; Prospective Studies Collaboration: Blood cholesterol and vascular mortality by age, sex, and blood pressure: a meta-analysis of individual data from 61 prospective studies with 55,000 vascular deaths. Lancet 370: 1829-1839, 2007.

8. Sassano A and Platanias LC: Statins in tumor suppression. Cancer Lett 260: 11-19, 2008.

9. Chan KK, Oza AM and Siu LL: The statins as anticancer agents. Clin Cancer Res 9: 10-19, 2003. 
10. Parikh A, Childress C, Deitrick K, Lin Q, Rukstalis D and Yang W: Statin-induced autophagy by inhibition of geranylgeranyl biosynthesis in prostate cancer PC3 cells. Prostate 70: 971-981, 2010

11. Schleicher SM, Moretti L, Varki V and Lu B: Progress in the unraveling of the endoplasmic reticulum stress/autophagy pathway and cancer: implications for future therapeutic approaches. Drug Resist Updat 13: 79-86, 2010.

12. Park WH, Kim ES, Kim BK and Lee YY: Monensin-mediated growth inhibition in NCI-H929 myeloma cells via cell cycle arrest and apoptosis. Int J Oncol 23: 197-204, 2003.

13. Robinson SM, Tsueng G, Sin J, et al: Coxsackievirus B exits the host cell in shed microvesicles displaying autophagosomal markers. PLoS Pathog 10: e1004045, 2014.

14. Gong JS and Kim GJ: The role of autophagy in the placenta as a regulator of cell death. Clin Exp Reprod Med 41: 97-107, 2014.

15. Hannigan AM and Gorski SM: Macroautophagy: the key ingredient to a healthy diet? Autophagy 5: 140-151, 2009.

16. Nishino I: Autophagic vacuolar myopathy. Semin Pediatr Neurol 13: 90-95, 2006.

17. Gozuacik D and Kimchi A: Autophagy and cell death. Curr Top Dev Biol 78: 217-245, 2007

18. Yang ZJ, Chee CE, Huang S and Sinicrope F: Autophagy modulation for cancer therapy. Cancer Biol Ther 11: 169-176, 2011

19. Yue Z, Jin S, Yang C, Levine AJ and Heintz N: Beclin 1, an autophagy gene essential for early embryonic development, is a haploinsufficient tumor suppressor. Proc Natl Acad Sci USA 100: 15077-15082, 2003.

20. Park MA, Zhang G, Martin AP, et al: Vorinostat and sorafenib increase ER stress, autophagy and apoptosis via ceramide-dependent CD95 and PERK activation. Cancer Bio Ther 7: 1648-1662, 2008.

21. Katayama M, Kawaguchi T, Berger MS and Pieper RO: DNA damaging agent-induced autophagy produces a cytoprotective adenosine triphosphate surge in malignant glioma cells. Cell Death Differ 14: 548-558, 2007.

22. Cao C, Subhawong T, Albert JM, et al: Inhibition of mammalian target of rapamycin or apoptotic pathway induces autophagy and radiosensitizes PTEN null prostate cancer cells. Cancer Res 66 : 10040-10047, 2006

23. Duffy A, Le J, Sausville E and Emadi A: Autophagy modulation: a target for cancer treatment development. Cancer Chemother Pharmacol: Nov 252014 (Epub ahead of print).

24. Song X, Liu H, Wang X, Li Z and Huang C: Atorvastatin combined with poly-unsaturated fatty acid confers better improvement of dyslipidemia and endothelium function. Lipids Health Dis 13: 186, 2014.

25. Pearson T, Ballantyne C, Sisk C, Shah A, Veltri E and Maccubbin D: Comparison of effects of ezetimibe/simvastatin versus simvastatin versus atorvastatin in reducing C-reactive protein and low-density lipoprotein cholesterol levels. Am J Cardiol 99: 1706-1713, 2007

26. Yang PM, Liu YL, Lin YC, Shun CT, Wu MS and Chen CC: Inhibition of autophagy enhances anticancer effects of atorvastatin in digestive malignancies. Cancer Res 70: 7699-7709, 2010.
27. Heymann D: Autophagy: A protective mechanism in response to stress and inflammation. Curr Opin Investig Drugs 7: 443-450, 2006.

28. He Z, Zhang Y, Mehta SK, Pierson DL, Wu H and Rohde LH: Expression profile of apoptosis related genes and radio-sensitivity of prostate cancer cells. J Radiat Res 52: 743-751, 2011.

29. Zhu K, Dunner K Jr and McConkey DJ: Proteasome inhibitors activate autophagy as a cytoprotective response in human prostate cancer cells. Oncogene 29: 451-462, 2010.

30. Umemiya-Shirafuji R, Matsuo T, Liao M, Boldbaatar D, Battur B, Suzuki H and Fujisaki K: Increased expression of ATG genes during nonfeeding periods in the tick Haemaphysalis longicornis. Autophagy 6: 473-481, 2010.

31. Aguilera MO, Berón W and Colombo MI: The actin cytoskeleton participates in the early events of autophagosome formation upon starvation induced autophagy. Autophagy 8: 1590-1603, 2012.

32. Levine B: Eating oneself and uninvited guests: autophagy-related pathways in cellular defense. Cell 120: 159-162, 2005.

33. Yu C, Wang L, Lv B, et al: TMEM74, a lysosome and autophagosome protein, regulates autophagy. Biochem Biophys Res Commun 369: 622-629, 2008.

34. Kirkin V, McEwan DG, Novak I and Dikic I: A role for ubiquitin in selective autophagy. Mol Cell 34: 259-269, 2009.

35. Behrends C, Sowa ME, Gygi SP and Harper JW: Network organization of the human autophagy system. Nature 466: 68-76, 2010.

36. Levine B and Yuan J: Autophagy in cell death: an innocent convict? J Clin Invest 115: 2679-2688, 2005.

37. Berry DL and Baehrecke EH: Autophagy functions in programmed cell death. Autophagy 4: 359-360, 2008.

38. Shimizu S, Kanaseki T, Mizushima N, Mizuta T, Arakawa-Kobayashi S, Thompson CB and Tsujimoto Y: Role of Bcl-2 family proteins in a non-apoptotic programmed cell death dependent on autophagy genes. Nat Cell Biol 6: 1221-1228, 2004.

39. Espert L, Denizot M, Grimaldi M, et al: Autophagy is involved in T cell death after binding of HIV-1 envelope proteins to CXCR4. J Clin Invest 116: 2161-2172, 2006

40. Chen ZH, Lam HC, Jin Y, et al: Autophagy protein microtubule-associated protein 1 light chain-3B (LC3B) activates extrinsic apoptosis during cigarette smoke-induced emphysema. Proc Natl Acad Sci USA 107: 18880-18885, 2010.

41. Weber JD, Jeffers JR, Rehg JE, et al: p53-independent functions of the p19(ARF) tumor suppressor. Genes Dev 14:2358-2365, 2000.

42. Matsuoka M, Kurita M, Sudo H, Mizumoto K, Nishimoto I and Ogata E: Multiple domains of the mouse p19ARF tumor suppressor are involved in p53-independent apoptosis. Biochem Biophys Res Commun 301: 1000-1010, 2003.

43. Paliwal S, Pande S, Kovi RC, Sharpless NE, Bardeesy N and Grossman SR: Targeting of C-terminal binding protein (CtBP) by ARF results in p53-independent apoptosis. Mol Cell Biol 26: 2360-2372, 2006.

44. Liang XH, Jackson S, Seaman M, Brown K, Kempkes B, Hibshoosh $\mathrm{H}$ and Levine B: Induction of autophagy and inhibition of tumorigenesis by beclin 1. Nature 402: 672-676, 1999 . 